\title{
Investigation of Various Cross-Linking Methods for the Immobilization of Cytosine Arabinoside on Bacterial Magnetosomes
}

\author{
Qinglei Dai, ${ }^{1}$ Yiming Ma, ${ }^{1}$ Shibin Wang, ${ }^{1,2,3}$ \\ Ranjith Kumar Kankala, ${ }^{1,3}$ and Yuangang Liu ${ }^{1,2,3}$ \\ ${ }^{1}$ College of Chemical Engineering, Huaqiao University, Xiamen 361021, China \\ ${ }^{2}$ Institute of Pharmaceutical Engineering, Huaqiao University, Xiamen 361021, China \\ ${ }^{3}$ Fujian Provincial Key Laboratory of Biochemical Technology, Xiamen 361021, China \\ Correspondence should be addressed to Yuangang Liu; ygliu@hqu.edu.cn
}

Received 16 March 2017; Revised 26 April 2017; Accepted 27 April 2017; Published 22 May 2017

Academic Editor: Vidyadhar Singh

Copyright ( 2017 Qinglei Dai et al. This is an open access article distributed under the Creative Commons Attribution License, which permits unrestricted use, distribution, and reproduction in any medium, provided the original work is properly cited.

Bacterial magnetosomes (BMs) have emerged as potential drug delivery vehicles, possessing an iron oxide or iron sulfide core surrounded by a natural lipid membrane shell. In this study, we immobilized cytosine arabinoside (Ara-C) effectively on BMs by using various methods such as direct absorption (ABMs), and others include different cross-linkers such as genipin (GP) and glutaraldehyde $(\mathrm{G})$. A well-dispersed Ara-C coupled bacterial magnetosomes resulted in significantly higher negative charge than that of naked BMs $(-11.5 \pm 0.3 \mathrm{mV})$ confirming the drug loading. Out of all methods, direct absorption process led to the highest encapsulation efficiency and drug loading of $88.2 \pm 4.3 \%$ and $46.9 \pm 1.2 \%$, respectively. These designs have shown the long-term drug release behavior without an initial burst release. Our results indicate that BMs-based nanoconjugates will potentially find widespread applications in pharmaceutical field.

\section{Introduction}

Recently, the explosive growth of utilization of nanotechnology for the preparation of delivery systems has led to the great progress in the field of medicine [1]. This technology provides an efficient alternative strategy to conventional delivery systems in not only for delivering drugs and genes to various therapeutic sites but also for bioimaging, tissue engineering, and others [2,3]. The efficient therapeutic strategies from this technology include, but are not limited to, polymer nanoparticles $[4,5]$, lipid-based nanoparticles $[6,7]$, and inorganic nanomaterials based on iron oxide [8], gold [9], and quantum dots and others [10]. Amongst inorganic nanomaterials, magnetic nanoparticles have drawn much attention in biomedical, medical diagnosis, and medical fields because of their tunable size, narrow size distribution, and unique optoelectronic features [11-13]. They have shown remarkable potential in biomedical research, especially their ability in combining therapeutic moieties with diagnostic agents $[14,15]$, which attributes to their inherent biocompatibility, magnetic properties, and high surface-area-to-volume ratio. Negatively charged bacterial magnetosomes (BMs) are one of the most efficient magnetic nanoparticles intended for the delivery of various bioactive moieties [16, 17]. In regard of designing the drug delivery carrier, BMs do not require any external modification/surface functionalization to improve the drug loading efficiency unlike that required for other magnetic nanoparticles. In general, BMs are biogenic magnetite derived from magnetotactic bacteria. These are composed of iron oxide or iron sulfide minerals enveloped by lipid membrane rich in amino groups $[18,19]$. In addition, previous literatures have evidenced that $\mathrm{BMs}$ are used to deliver various therapeutic agents such as drugs [20, 21], enzyme [22], and DNA [23]. In a case, the activity of glucose oxidase immobilized BMs is 40 times effective than those immobilized on artificial magnetite particles [24]. In 


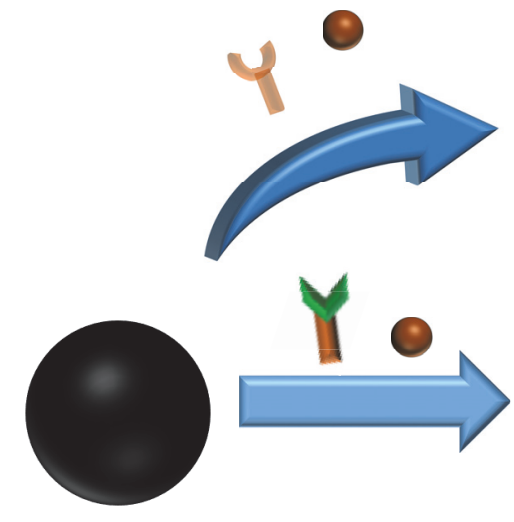

BMs

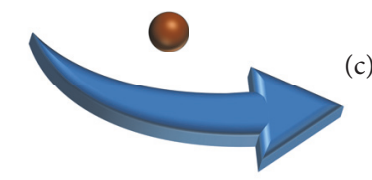

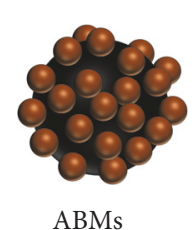

ABMs

(a)

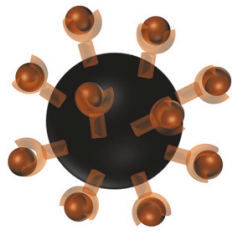

ABMs-GP

(b)

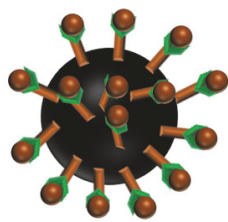

ABMs-G

$-\mathrm{GP} O$-Ara-C
$-\mathrm{G}$

Scheme 1: Graphical illustration showing the Ara-C immobilization on BMs through various methods and their resultant end products, (a) genipin (GP) cross-linker (ABMs-GP), (b) glutaraldehyde (G) cross-linker (ABMs-G), and (c) direct absorption method (ABMs).

another instance, BMs have been used as vectors for the chemotherapeutic drug conjugation, that is, methotrexate (MTX), which has shown exceptional loading and in vitro release behavior of MTX [25]. Moreover, after modifying with poly-L-glutamic acid, the drug loading amount was significantly increased in BMs [26].

In this paper, we designed a delivery system possessing BMs loaded with Ara-C effectively by using different methods such as direct absorption (ABMs), and others include different cross-linkers (GP and $G$ ) which resulted in different ABMs (ABMs-GP, ABMs-G), respectively (Scheme 1). Further, we investigate the release behavior of antitumor drug Ara-C from BMs nanoconjugates.

\section{Materials and Methods}

2.1. Materials. Bacterial magnetosomes derived from Magnetospirillum sp. ME-1 were provided by Wuhan Institute of Virology, Chinese Academy of Sciences. Ara-C was purchased from Sunray Pharmaceutical Co., Ltd. (Suzhou, China). Genipin (GP) was obtained from Zhixin Biotechnology Company (Fuzhou, China). Glutaraldehyde (G) was purchased from Sigma Aldrich (USA). All other additional chemicals utilized were purchased from Sinopharm Chemical Reagent Co., Ltd. (Beijing, China).
2.2. Ara-C Immobilization on BMs. Due to abundant amino groups in the lipid membrane shell, bacterial magnetosomes isolated from the cells of magnetotactic bacteria are extremely suitable for Ara-C immobilization [19]. Ara-C also has the amino active sites suitable for immobilization. The immobilization of Ara-C is investigated by using various methods such as direct absorption method, and others include various cross-linkers (GP and G). The scheme of preparation was based on our previous study [27].

Initially, purified BMs were suspended in PBS ( $\mathrm{pH} 7.4$ ) and the suspension was subjected to ultrasonic bathing at $50 \mathrm{~W}$ for $5 \mathrm{~min}$ to distribute the BMs adequately. BMs were then incubated with Ara-C by sonication ( $50 \mathrm{~W}, 5 \mathrm{~min}$ ). Following the addition of GP solution $(0.5 \%)$, the mixture was distributed by ultrasonic bathing for ten times $(50 \mathrm{~W}$, each lasted for $1 \mathrm{~min}$ with an interval of $5 \mathrm{~min}$ ). The mixture was then placed in an incubator shaker with provided conditions $\left(60 \mathrm{rpm}, 37^{\circ} \mathrm{C}\right.$ for $\left.72 \mathrm{~h}\right)$. The resultant ABMs-GP was derived from the blend by magnet and then washed carefully with PBS ( $\mathrm{pH}$ 7.4). Other designs such as ABMs-G and ABMs were obtained through the same above discussed process, but the only change is by using $\mathrm{G}$ and no cross-linker, respectively.

2.3. Physical Characterization. Transmission electron microscope (TEM) images were captured following the procedure 
TABLE 1: Zeta potentials of BMs, ABMs-GP, ABMs-G, and ABMs.

\begin{tabular}{lcccc}
\hline Sample & BMs & ABMs-GP & ABMs-G & ABMs \\
\hline Zeta potential $(\mathrm{mV})$ & $-11.5 \pm 0.3$ & $-29.1 \pm 1.7$ & $-34.6 \pm 3.0$ & $-35.9 \pm 2.7$ \\
\hline
\end{tabular}

for preparing samples. The prepared complexes were washed with PBS and distilled water for many times, followed by being suspended in distilled water using an ultrasonic bathing. Further, the BMs and the previous complex supernatants $(15-20 \mu \mathrm{L})$ were dropped on the cropper grids. After being dried, all grids were observed under the TEM. In addition, surface charge measurements were performed using Zetasizer. The samples were suspended in water, and $1 \mathrm{~mL}$ of suspension was subjected to record the surface charge using Zetasizer analyser (ZEN 3600; Malvern Instruments, Ltd., Malvern, UK). All assays were performed in triplicate, and the DTS5.00 for windows was used for statistical analysis.

2.4. Examination by FTIR Spectrometer. To elucidate the functional groups attached, Fourier transform infrared (FTIR) spectra were recorded on NICOLET iS10, Thermo Fisher Scientific spectrometer at a scanning range of $4000-400 \mathrm{~cm}^{-1}$. Samples were prepared using a dried $\mathrm{KBr}$ pellet method.

2.5. Evaluation of the Amount of Ara-C Immobilized to $B M s$. Appropriate sample suspension was taken out and dissolved in $4.8 \mathrm{~mL}$ breaking-membrane liquid composed of $37 \%$ hydrochloric acid and $70 \%$ ethanol solution $(\mathrm{v} / \mathrm{v}=1: 2)$ for $1 \mathrm{~h}$. The number of Ara-C loaded with BMs was calculated based on a standard curve for Ara-C in breaking-membrane liquid: $Y=24.4764 X+0.3134\left(R^{2}=0.9994\right)$, where $Y$ is the concentration of Ara-C $(\mu \mathrm{g} / \mathrm{mL})$ and $X$ is the absorbance of Ara-C in membrane-breaking liquid measured at $283.5 \mathrm{~nm}$.

2.6. In Vitro Drug Release Study. The tests of Ara-C released from various samples were performed by placing the sample containing tubes in an incubator shaker at $60 \mathrm{rpm}, 37^{\circ} \mathrm{C}$ with magnet absorption. The resultants were diluted into PBS at $\mathrm{pH} 7.4$ and incubated for $0.5,1,12,24,36$, or $48 \mathrm{~h}$. At settled time points, the absorption value of Ara-C in the supernatant was measured at $271.4 \mathrm{~nm}$ using $\mathrm{U}$-vis spectrophotometer. The concentration of Ara-C was determined using a standard curve: $Y=26.0769 X-0.0625\left(R^{2}=0.9999\right)$, where $Y$ is the concentration of Ara-C $(\mu \mathrm{g} / \mathrm{mL})$ and $X$ is the absorption of Ara-C in PBS at $271.4 \mathrm{~nm}$. All assays were performed in triplicate.

\section{Results and Discussion}

3.1. Immobilization of Ara-C on BMs. The commonly used method to prepare BMs-based conjugates was through amine-amine linkage [28]. We investigated various approaches to couple Ara-C with BMs using different crosslinkers. Figure 1 depicts the TEM image observations elucidating the surface morphology of BMs-based conjugates. A distinct membrane outside of BMs is clearly visible and it is noteworthy that various conjugates (Figures 1(b)-1(d)) look like they were covered by thickened and blurred materials, which is absent in BMs (Figure 1(a)). This infers that Ara-C is loaded onto the surface of BMs.

The surface charge of nanoconjugates plays a key role in elucidating the drug loading efficiency. As shown in Table 1, the zeta potential values of all drug conjugates were significantly lower than that of BMs $(-11.5 \pm 0.3 \mathrm{mV})$. The changes indicate that Ara-C is successfully loaded onto the BMs. This phenomenon could be the occupying of positively charged groups in the membrane. The results also show that the resultant conjugates are stable in aqueous solution.

3.2. Examination by FTIR Spectrometer. FTIR spectra were recorded to characterize the chemical groups and surface modifications within the modified samples. The FTIR spectra of Ara-C, ABMs-GP, ABMs-G, ABMs, and BMs samples are shown in Figure 2. The main characteristic absorption peaks of BMs were at $3435,2925,1724,1635,1563,1057$, and $589 \mathrm{~cm}^{-1}$. The $\mathrm{NH}_{2}$ stretching vibration should appear at 3435 and $1563 \mathrm{~cm}^{-1}$. The peaks at 1724 and $1635 \mathrm{~cm}^{-1}$ were assigned to $\mathrm{C}=\mathrm{O}$ and $\mathrm{C}=\mathrm{C}$ stretching vibration. The absorption peaks observed at $1057 \mathrm{~cm}^{-1}$ represents the phosphate stretching vibration signals. The weak absorption peak at $589 \mathrm{~cm}^{-1}$ was assigned to the typical $\mathrm{Fe}-\mathrm{O}$ stretching vibration [29].

It is notable that the FTIR of ABMs-GP showed weaker absorbance at both 3435 and $1568 \mathrm{~cm}^{-1}$ than that of BMs, due to the reduction of amino groups on ABMs-GP, while the stronger absorbance peak at $1728 \mathrm{~cm}^{-1}$ also was found, which represents the introduction of carbonyl groups [21]. The absorption peak at $809 \mathrm{~cm}^{-1}$ assigned to Ara-C was found in ABMs-GP. Additionally, the FTIR of ABMs-G showed stronger absorbance peak at 1059 and $1634 \mathrm{~cm}^{-1}$. Moreover, contrary to the BMs, both the FTIR of ABMs and Ara-C showed absorbance peak at $809 \mathrm{~cm}^{-1}$. All these data manifested that Ara-C is loaded on to BMs membrane via different cross-linkers.

3.3. Evaluation of the Amount of Ara-C Immobilized to BMs. It is well known that high coupling efficiency can facilitate the therapy. Figure 3 depicts that the amount of Ara-C coupled to BMs changes over cross-linking methods. The drug loading of ABMs is much higher than that of previous study [30]. The high drug loading may be due to the abundant amine groups and narrow size distribution. Most important is that direct absorption methods exhibited the highest coupling efficacy and encapsulation efficiency within the experiment, owing to the high surface to volume ratio of BMs [31]. Nevertheless, the drug loading of ABMs-GP and ABMs-G by covalent cross-linking is lower than that of ABMs; it may be because the reaction process is affected by various other factors such as $\mathrm{pH}$, temperature, and concentration. In this paper, we 


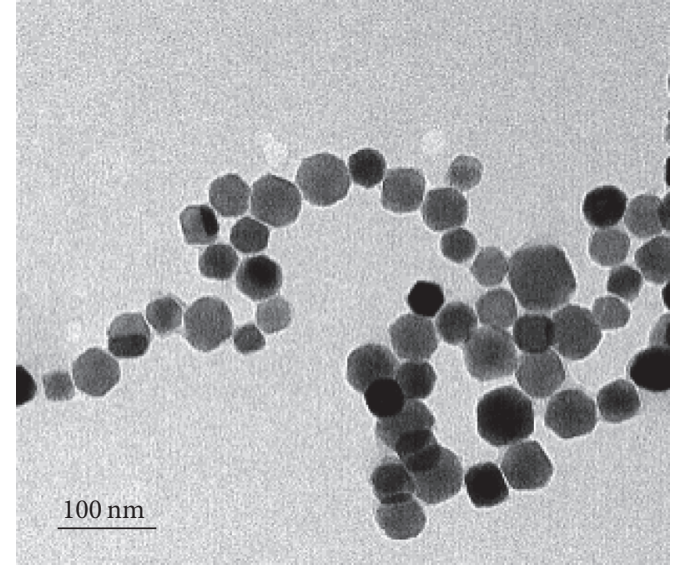

(a)

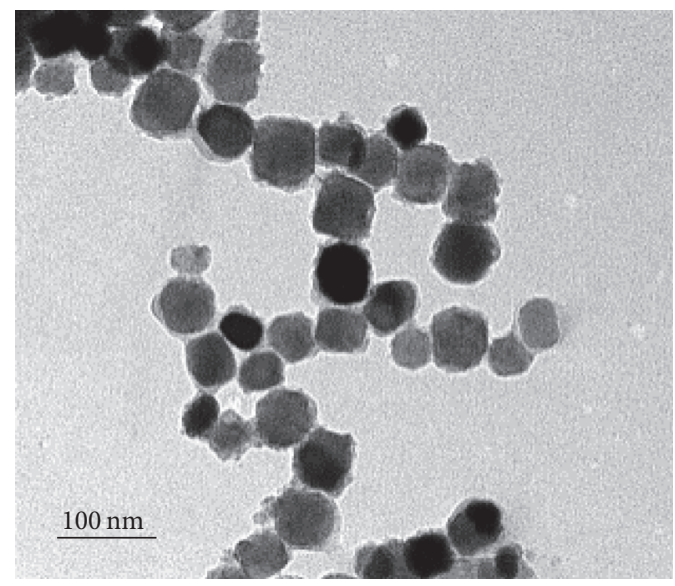

(c)

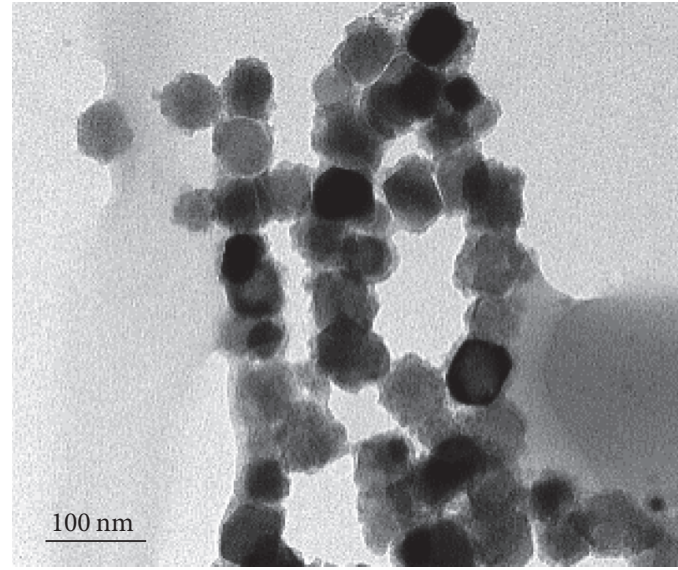

(b)

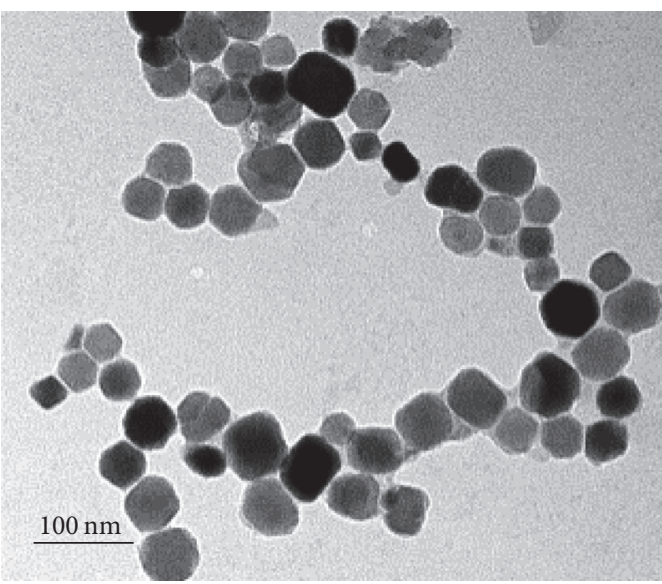

(d)

Figure 1: TEM images of BMs (a), ABMs-GP (b), ABMs-G (c), and ABMs (d).

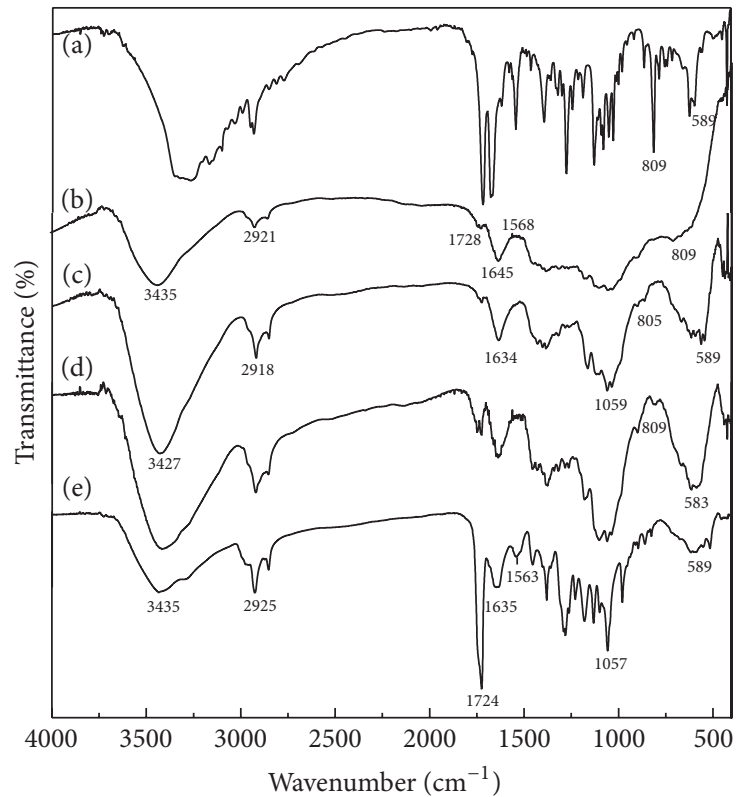

Figure 2: The FTIR spectra of (a) Ara-C, (b) ABMs-GP, (c) ABMs-G, (d) ABMs, and (e) BMs. 


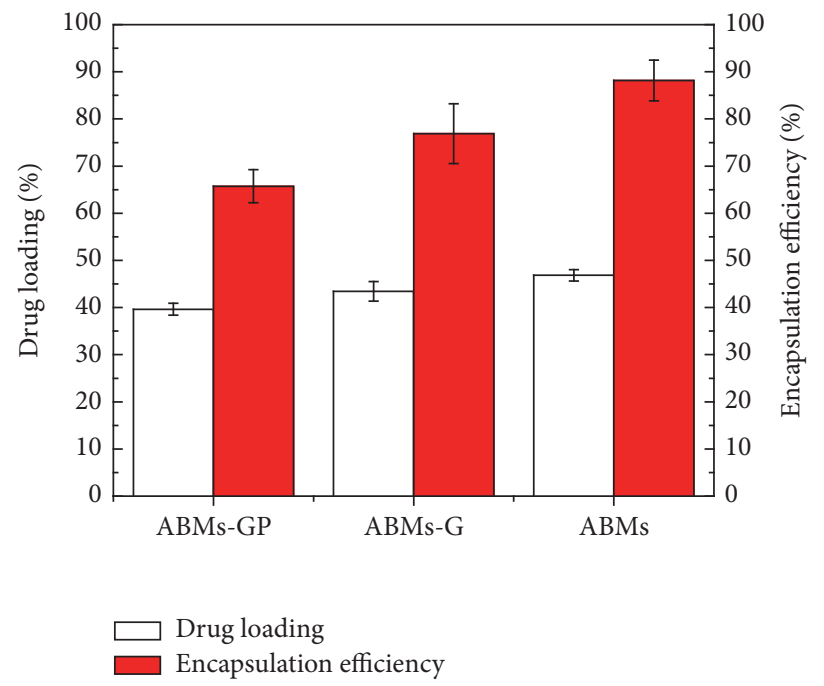

FIGURE 3: Drug loading and encapsulation efficiency of ABMs-GP, ABMs-G, and ABMs.

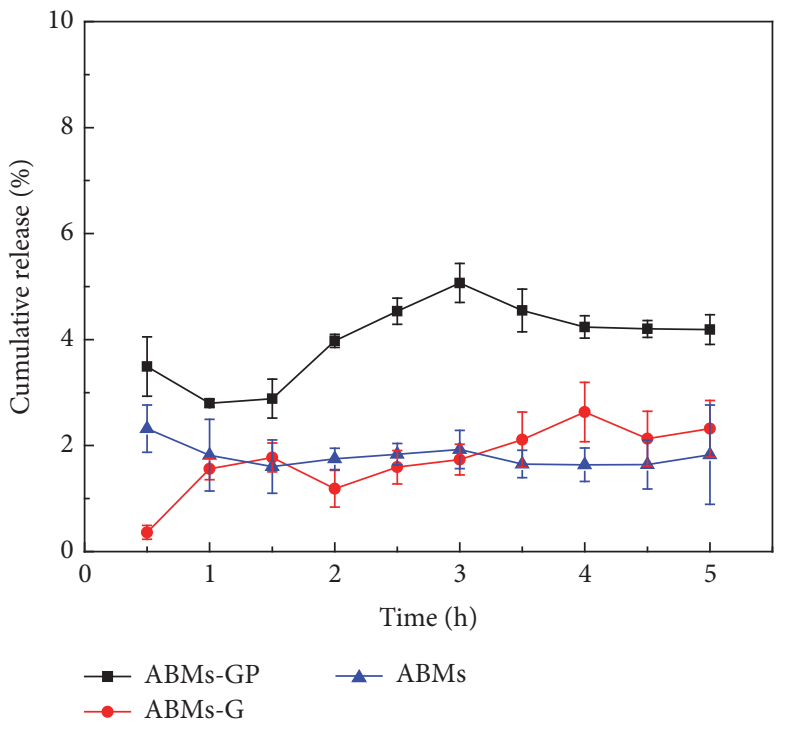

(a)

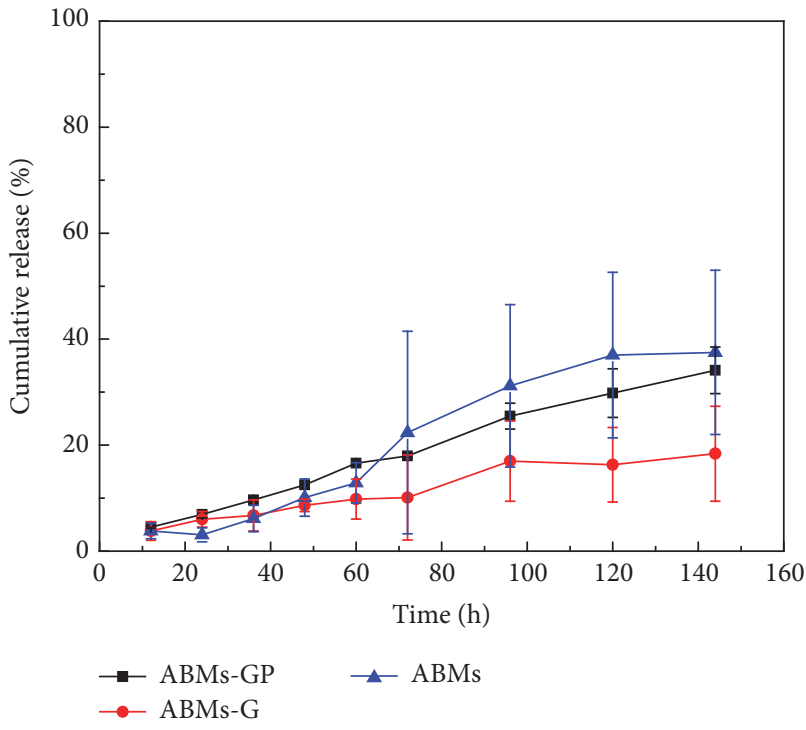

(b)

FIGURE 4: The cumulative release of compounds with different reparation methods in vitro (a) before 5 hours and (b) after 5 hours.

selected the optimum condition of our previous study [20]. The other factor is the steric effect of intermolecular reaction, which inhibits the experiment process. The results of drug loading are consistent with that of zeta potentials.

3.4. In Vitro Drug Release. The release of Ara-C from BMsbased conjugates was assayed by suspending them in PBS (pH 7.4) to mimic pH of blood plasma [32]. As shown in Figure 4, drug burst release behavior was not observed in the initial half an hour. Ara-C releases slowly and lower than $40 \%$ of the original drug after incubation for $140 \mathrm{~h}$. This phenomenon indicates that BMs-based conjugates are endowed with a long-term release property. Ara-C was released more readily from ABMs-G than that of ABMs-GP and ABMs (Figure 4(b)). The reason may be attributed to the fact that intermolecular forces of direct absorption are weaker than covalent cross-linking. However, at the first 5 hours (Figure 4(a)), the cumulative release of ABMs was a little slower, which may be a compromise result of the highest drug loading and the relatively higher release amount.

\section{Conclusions}

In summary, Ara- $\mathrm{C}$ was immobilized on BMs efficiently by using GP, G, and direct absorption. Direct absorption shows the highest drug loading of $46.9 \pm 1.2 \%$. In comparison with different conjugates, $\mathrm{ABMs}$ shows better dispersion and much more stable long-term release property. Our study provides a theoretical basis for cross-linking method to reduce the amount of drug and improve the therapy efficiency. 


\section{Conflicts of Interest}

The authors declare that they have no conflicts of interest.

\section{Acknowledgments}

Financial support from NSFC (31000441 and 31170939), National Marine Economic Innovation and Development Project (16PYY007SF17), the Science Research Foundation of National Health and Family Planning Commission of PRC \& United Fujian Provincial Health and Education Project for Tacking the Key Research (WKJ2016-2-22), the Program for New Century Excellent Talents in Fujian Province University (2014FJ-NCET-ZR01), and the Promotion Program for Young and Middle-Aged Teachers in Science and Technology Research of Huaqiao University (ZQN-PY108) is gratefully acknowledged.

\section{References}

[1] X. Y. Zhang and P. Y. Zhang, "Nanotechnology for multimodality treatment of cancer," Oncology Letters, vol. 12, pp. 4883-4886, 2016.

[2] J. Hu, Y. Tang, A. H. Elmenoufy, H. Xu, Z. Cheng, and X. Yang, "Nanocomposite-based photodynamic therapy strategies for deep tumor treatment," Small, vol. 11, no. 44, pp. 5860-5887, 2015.

[3] A. Wicki, D. Witzigmann, V. Balasubramanian, and J. Huwyler, "Nanomedicine in cancer therapy: challenges, opportunities, and clinical applications," Journal of Controlled Release, vol. 200, pp. 138-157, 2015.

[4] R. K. Kankala, Y. Kuthati, C. L. Liu et al., "Killing cancer cells by delivering a nanoreactor for inhibition of catalase and catalytically enhancing intracellular levels of ROS," RSC Advances, vol. 5, no. 105, pp. 86072-86081, 2015.

[5] N. Morimoto, M. Wakamura, K. Muramatsu et al., "Membrane translocation and organelle-selective delivery steered by polymeric zwitterionic nanospheres," Biomacromolecules, vol. 17, no. 4, pp. 1523-1535, 2016.

[6] D. Colmenares, Q. Sun, P. Shen, Y. Yue, D. J. McClements, and Y. Park, "Delivery of dietary triglycerides to caenorhabditis elegans using lipid nanoparticles: nanoemulsion-based delivery systems," Food Chemistry, vol. 202, pp. 451-457, 2016.

[7] P. S. Apaolaza, A. del Pozo-Rodríguez, M. A. Solinís et al., "Structural recovery of the retina in a retinoschisin-deficient mouse after gene replacement therapy by solid lipid nanoparticles," Biomaterials, vol. 90, pp. 40-49, 2016.

[8] L. Rodriguez-Arco, I. A. Rodriguez, V. Carriel et al., "Biocompatible magnetic core-shell nanocomposites for engineered magnetic tissues," Nanoscale, vol. 8, no. 15, pp. 8138-8150, 2016.

[9] S. Yook, Y. Lu, J. J. Jeong et al., "Stability and biodistribution of thiol-functionalized and 177lu-labeled metal chelating polymers bound to gold nanoparticles," Biomacromolecules, vol. 17, no. 4, pp. 1292-1302, 2016.

[10] T. Song, H. Cheng, C. Fu et al., "Influence of the active layer nanomorphology on device performance for ternary PbSxSe1$\mathrm{x}$ quantum dots based solution-processed infrared photodetector," Nanotechnology, vol. 27, no. 16, Article ID 165202, 2016.

[11] S. Chen, X. Hao, X. Liang et al., "Inorganic nanomaterials as carriers for drug delivery," Journal of Biomedical Nanotechnology, vol. 12, no. 1, pp. 1-27, 2016.
[12] X. Li, J. Wei, K. E. Aifantis et al., "Current investigations into magnetic nanoparticles for biomedical applications," Journal of Biomedical Materials Research Part A, vol. 104, no. 5, pp. 12851296, 2016.

[13] H. Liu, J. Zhang, X. Chen et al., "Application of iron oxide nanoparticles in glioma imaging and therapy: from bench to bedside," Nanoscale, vol. 8, no. 15, pp. 7808-7826, 2016.

[14] S. Wang, W. T. Yang, H. L. Du et al., "Multifunctional reductionresponsive SPIO\&DOX-loaded PEGylated polymeric lipid vesicles for magnetic resonance imaging-guided drug delivery," Nanotechnology, vol. 27, no. 16, Article ID 165101, 2016.

[15] G. Lin, W. Zhu, L. Yang et al., "Delivery of siRNA by MRIvisible nanovehicles to overcome drug resistance in MCF7/ADR human breast cancer cells," Biomaterials, vol. 35, no. 35, pp. 9495-9507, 2014.

[16] M. Boucher, F. Geffroy, S. Prévéral et al., "Genetically tailored magnetosomes used as MRI probe for molecular imaging of brain tumor," Biomaterials, vol. 121, pp. 167-178, 2017.

[17] C. Chen, P. Wang, and L. Li, "Applications of bacterial magnetic nanoparticles in nanobiotechnology," Journal of Nanoscience and Nanotechnology, vol. 16, no. 3, pp. 2164-2171, 2016.

[18] A. C. V. Araujo, F. Abreu, K. T. Silva, D. A. Bazylinski, and U. Lins, "Magnetotactic bacteria as potential sources of bioproducts," Marine Drugs, vol. 13, no. 1, pp. 389-430, 2015.

[19] A. S. Mathuriya, "Magnetotactic bacteria for cancer therapy," Biotechnology Letters, vol. 37, no. 3, pp. 491-498, 2015.

[20] Y.-G. Liu, Q.-L. Dai, S.-B. Wang, Q.-J. Deng, W.-G. Wu, and A.-Z. Chen, "Preparation and in vitro antitumor effects of cytosine arabinoside-loaded genipin-poly-L-glutamic acidmodified bacterial magnetosomes," International Journal of Nanomedicine, vol. 10, pp. 1387-1397, 2015.

[21] Q. Deng, Y. Liu, S. Wang et al., "Construction of a novel magnetic targeting anti-tumor drug delivery system: cytosine arabinoside-loaded bacterial magnetosome," Materials, vol. 6, no. 9, pp. 3755-3763, 2013.

[22] N. Nakamura, K. Hashimoto, and T. Matsunaga, "Immunoassay method for the determination of immunoglobulin $G$ using bacterial magnetic particles," Analytical Chemistry, vol. 63, no. 3, pp. 268-272, 1991.

[23] L. Xiang, W. Bin, J. Huali et al., "Bacterial magnetic particles (BMPs)-PEI as a novel and efficient non-viral gene delivery system," Journal of Gene Medicine, vol. 9, no. 8, pp. 679-690, 2007.

[24] T. Matsunaga and S. Kamiya, "Use of magnetic particles isolated from magnetotactic bacteria for enzyme immobilization," Applied Microbiology and Biotechnology, vol. 26, no. 4, pp. 328332, 1987.

[25] Y. Liu, M. Xie, S. Wang, Q. Zheng, A. Chen, and Q. Deng, "Facile fabrication of high performances MTX nanocomposites with natural biomembrane bacterial nanoparticles using GP," Materials Letters, vol. 100, pp. 248-251, 2013.

[26] L. Guo, J. Huang, and L.-M. Zheng, "Control generating of bacterial magnetic nanoparticle-doxorubicin conjugates by poly-L-glutamic acid surface modification," Nanotechnology, vol. 22, no. 17, Article ID 175102, 2011.

[27] R. M. Long, Y. G. Liu, Q. L. Dai et al., "A natural bacteriumproduced membrane-bound nanocarrier for drug combination therapy," Materials, vol. 9, no. 11, p. 889, 2016.

[28] J. Sun, Y. Li, X.-J. Liang, and P. C. Wang, "Bacterial magnetosome: a novel biogenetic magnetic targeted drug carrier with potential multifunctions," Journal of Nanomaterials, vol. 2011, Article ID 469031, 13 pages, 2011. 
[29] J.-B. Sun, J.-H. Duan, S.-L. Dai et al., "Preparation and antitumor efficiency evaluation of doxorubicin-loaded bacterial magnetosomes: magnetic nanoparticles as drug carriers isolated from Magnetospirillum gryphiswaldense," Biotechnology and Bioengineering, vol. 101, no. 6, pp. 1313-1320, 2008.

[30] L. Guo, Ji Huang, X. Zhang, Y. Li, and L. Zheng, "Bacterial magnetic nanoparticles as drug carriers," Journal of Materials Chemistry, vol. 18, no. 48, pp. 5993-5997, 2008.

[31] C. F. Chen, P. P. Wang, and L. L. Li, "Applications of bacterial magnetic nanoparticles in nanobiotechnology," Journal of Nanoscience \& Nanotechnology, vol. 16, no. 3, pp. 2164-2171, 2016.

[32] M. F. Wang, T. X. Liu, L. Q. Han et al., "Functionalized O-carboxymethyl-chitosan/polyethylenimine based novel dual $\mathrm{pH}$-responsive nanocarriers for controlled co-delivery of DOX and genes," Polymer Chemistry, vol. 6, no. 17, pp. 3324-3335, 2015. 

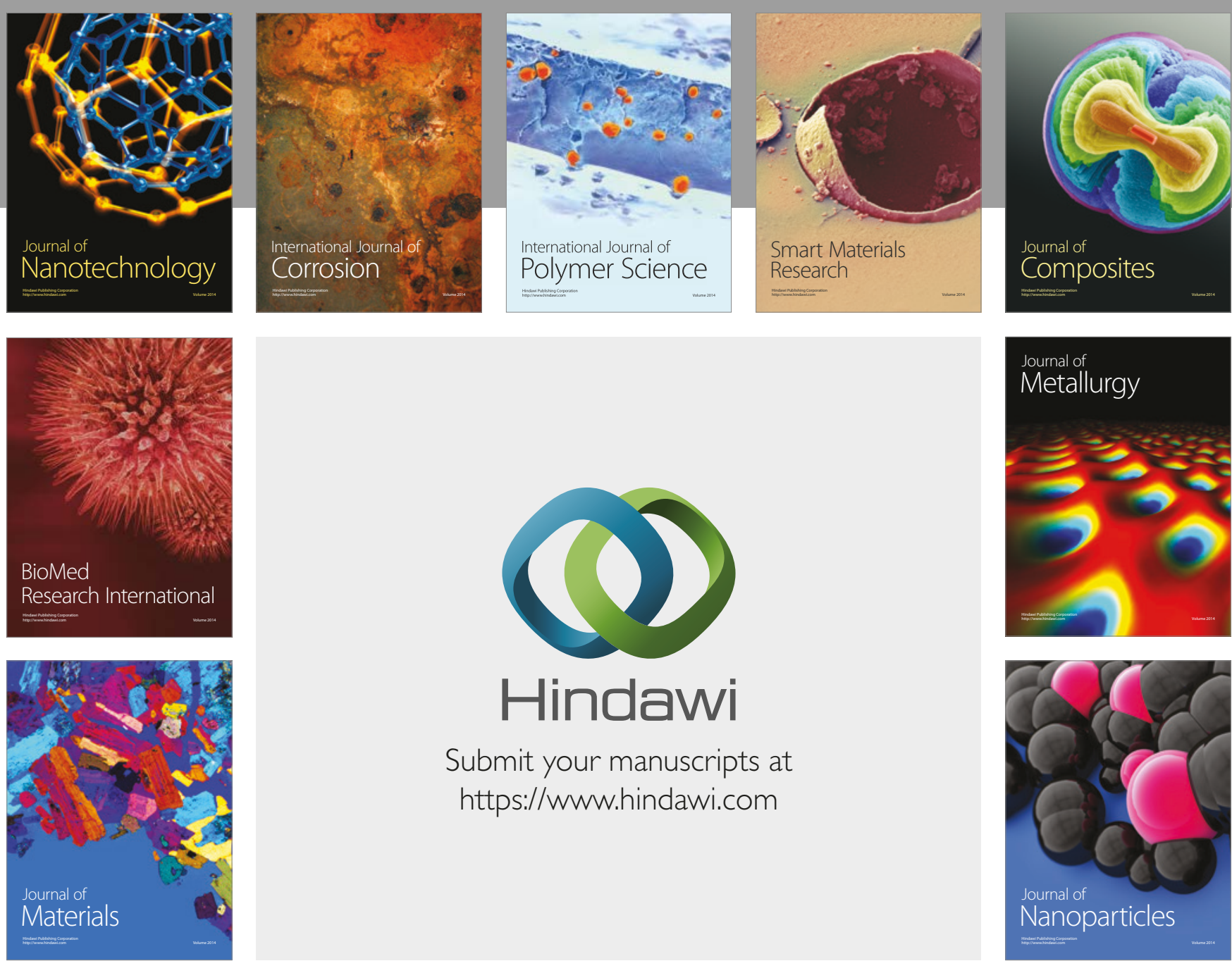

\section{Hindawi}

Submit your manuscripts at

https://www.hindawi.com
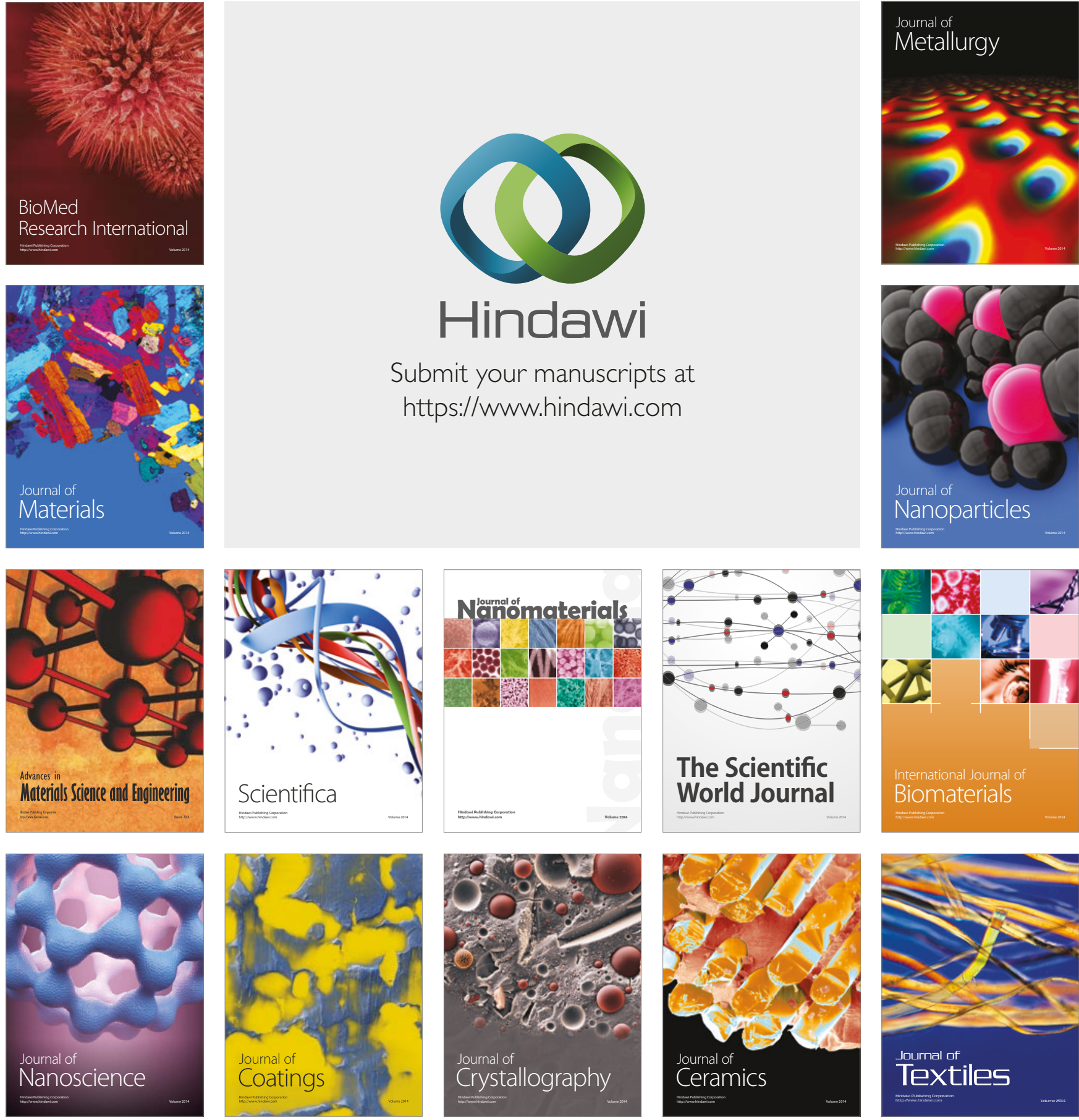

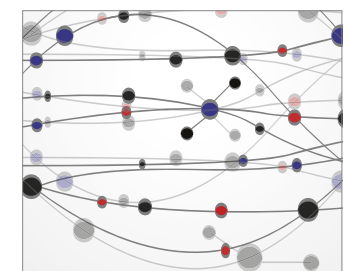

The Scientific World Journal
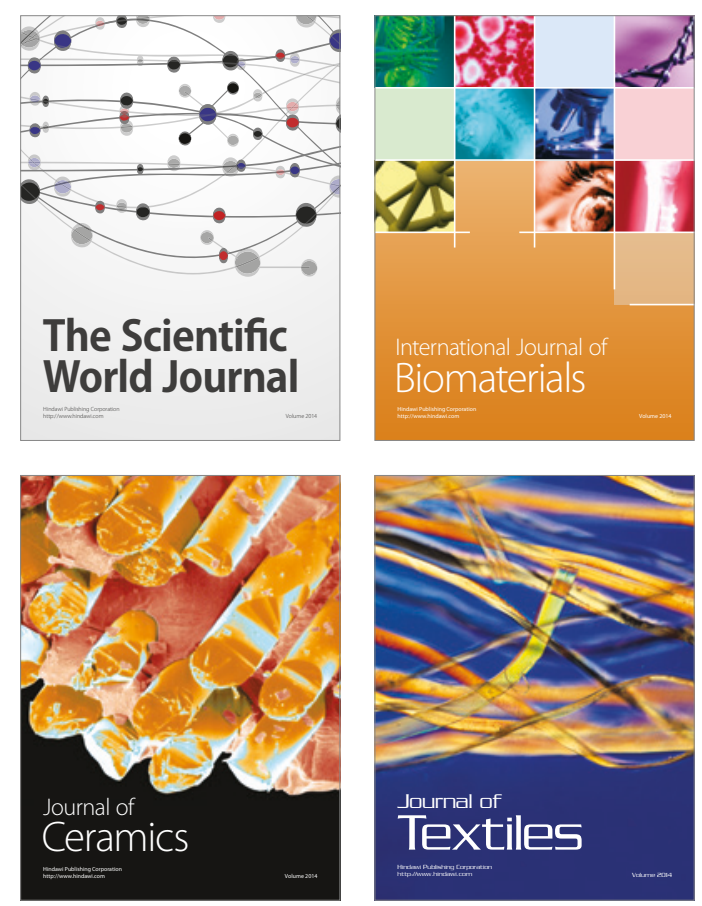